\title{
Robust Edge Detection Using Mumford-Shah Model and Binary Level Set Method
}

\author{
Li-Lian Wang ${ }^{1}$, Yuying $\mathrm{Shi}^{2}$, and Xue-Cheng Tai ${ }^{3}$ \\ 1 Division of Mathematical Sciences, School of Physical and Mathematical Sciences, \\ Nanyang Technological University, 637371, Singapore \\ 2 Department of Mathematics and Physics, North China Electric Power University, \\ Beijing, 102206 China \\ 3 Division of Mathematical Sciences, School of Physical and Mathematical Sciences, \\ Nanyang Technological University, 637371, Singapore, and Department of \\ Mathematics, University of Bergen, Bergen, Norway
}

\begin{abstract}
A new approximation of the Mumford-Shah model is proposed for edge detection, which could handle open-ended curves and closed curves as well. The essential idea is to treat the curves by narrow regions, and use a sharp interface technique to solve the approximate Mumford-Shah model. A fast algorithm based on the augmented Lagrangian method is developed. Numerical results show that the proposed model and method are very efficient and have the potential to be used for edge detections for real complicated images.
\end{abstract}

\section{Introduction}

Edge detection is one of the fundamental problems for image processing and computer vision [29], and its application also spans many other areas such as boundary extraction and solid-liquid interface detection $39 \mid 8]$ in material science and physics. In the context of image processing, edge detection is to find the boundaries of objects in a digital image. Many methods have been developed for this purpose. Classical approaches attempted to detect edges by discontinuities in image intensity values. Witkin 43 proposed the scale space framework to analyze images, where the extreme of the first gradient and zero-crossing of the second gradient are used to detect edges. However, the edges are usually disconnected, so Canny 11 proposed the so-called Canny edge detector to detect disconnected edges. The main principle is to classify a pixel into the edges, if the gradient magnitude of the pixel is larger than those of pixels at both sides in the image domain. The Canny's edge detector inspired many subsequential works, e.g., Susan 33 and Canny-Deriche [18].

Another important class of methods are based on nonlinear isotropic diffusive equations [13|1|30. In general, such models are designed to prevent smoothing near the edges and to encourage diffusion in the homogeneous regions. However, they may break down when the gradient generated by noise is comparable to the targeted edges. Along this line, some reaction and diffusion equations [35]42,

A.M. Bruckstein et al. (Eds.): SSVM 2011, LNCS 6667, pp. 291-301 2011.

(C) Springer-Verlag Berlin Heidelberg 2011 
edge flow [24, snake/active contour models 2011215] and universal gravity 34] are also proposed for edge detection.

It is obviously impossible to summarize all the methods and the interested readers are referred to $10|25| 23 \mid 31$ for a review of many other methods. Given the vast amount of existing algorithms, we feel compelled to provide sufficient justification for developing yet another method. Motivated by the success of piecewise constant level set methods for image segmentation 22] and the recent developed fast algorithms [6], we shall introduce a new edge detector based on a robust binary level set method for the Mumford-Shah model [27. In contrast with image segmentation, which is to find a partition of the image domain, edge detection aims more at finding the discontinuities of the intensity function. Hence, we are mostly concerned with the determination of open curves. However, there is no a natural way to represent open curves, since there is no distinction of interior and exterior regions. Accordingly, the standard level set methods [28] can not be directly applied. Among very few approaches for dealing with open curves, the work of 32 for modeling spiral crystal growth used two level set functions to represent the codimension-two boundary of the open curve by $\{x: \phi(x)=0$ and $\psi(x)>0\}$, where $\phi, \psi$ are two signed distance function. In [7, an open-ended curve was represented by the "centerline" of the level set function defined on the curve, and the motion of the curve was essentially driven by the evolution a small region surrounding the curve. Interestingly, a recent work of Leung and Zhao [21] proposed a grid based particle method to represent an open curve by the most relevant points in the neighborhood on the discrete grids. The authors also commented on the limitations of the methods in 327] for open curve evolution.

In this paper, we embed an open (or a closed) curve of interest in a narrow region (or band) with the curve being part of the (one-sided) boundary (see Figure 1 below for an illustration). From geometric point of view, such a region is formed by the parallel curve (also known as the offset curve) [38. We define a binary piecewise constant level set function on the small region, and show that the total variation of the level set function gives a good approximation of the length of the curve. Moreover, we add intrinsic forces to enforce the level set function to converge to binary values, and this helps to enhance and sharpen the edges. Moreover, applying this notion to translate the Mumford-Shah model leads to fast and robust algorithms for edge detection. On the other hand, we simultaneously solve the edge set and the optimal piecewise smooth approximation of the given image in the Mumford-Shah model, so the proposed methods can be used for mutliphase piecewise smooth image segmentation (which is yet a challenging topic [40]).

The rest of the paper is organized as follows. In Section 2, we formulate the Mumford-Shah model based on an embedding of the curve in a narrow band and the binary piecewise constant level set method. In Section 3, we describe the fast algorithm based on the augmented Lagrangian method. Section 4 is devoted to the numerical experiments for detection of open curves and segmentation of multiphase piecewise smooth images. Some concluding remarks are given in Section 5. 


\section{Formulation of the Model}

Given an image $I$ on an open bounded domain $\Omega \in \mathbb{R}^{2}$, Mumford and Shah in their seminal paper [27] suggested minimizing the following functional to find a piecewise smooth approximation $u$ of $I$ and the edge set $\Gamma$ :

$$
E(u, \Gamma)=\frac{\alpha}{2} \int_{\Omega}(u-I)^{2} d \boldsymbol{x}+\frac{\beta}{2} \int_{\Omega \backslash \Gamma}|\nabla u|^{2} d \boldsymbol{x}+|\Gamma|,
$$

where $|\Gamma|$ is the length of the edges picked from all over the image domain, and $\alpha, \beta$ are positive parameters to balance three terms. They also conjectured that $E$ has a minimizer and the edges (the discontinuity $\Gamma$ ) are the union of a finite set of $C^{1,1}$ embedded curves with three possible configurations [4: (i) a crap tip (look like a half-line or a single arc ends without meeting others); (ii) a triple junction (three curves meeting at their endpoints with $2 \pi / 3$ angle between each pair); and (iii) boundary points (a curve meets $\partial \Omega$ perpendicularly). The MumfordShah model and conjecture have inspired deep mathematical investigation and extensive applications 4 177. The reduced model (without the second term called the piecewise constant Mumford-Shah model), together with an appropriate level set implementation, becomes a fundamental tool for piecewise constant image segmentation (see, e.g., [15/22]). We are interested in detecting $\Gamma$ by solving the full model (11). The first issue is to characterize the edge set. An important idea is to associate $\Gamma$ with the jump $\Gamma_{u}$ of the unknown $u$, and this leads to the remarkable approximation of (10) by Ambrosio and Tortorelli [2]3]:

$$
E_{\varepsilon}(u, \Gamma)=\frac{\alpha}{2} \int_{\Omega}(u-I)^{2} d \boldsymbol{x}+\frac{\beta}{2} \int_{\Omega} v^{2}|\nabla u|^{2} d \boldsymbol{x}+\int_{\Omega}\left(\varepsilon|\nabla v|^{2}+\frac{1}{4 \varepsilon}(v-1)^{2}\right) d \boldsymbol{x},
$$

where $v$ is an auxiliary variable such that $v \approx 0$ if $x \in \Gamma_{u}$, and $v \approx 1$ otherwise. A rigorous analysis (see [2]9]) shows that the last term converges to $|\Gamma|$ in Gammaconvergence sense [16]. The width of transition from $v=0$ to 1 is about $O(\varepsilon)$.

In what follows, we take a different point of view to characterize $\Gamma$ and approximate its length. Based on Mumford and Shah conjecture, we assume that the targeted edge set $\Gamma$ consists of a finite union of simple (i.e., non-self-intersecting) curves with suitable regularity. Let $\boldsymbol{r}$ be a single curve in the edge set $\Gamma$ with a definite parameterization: $\boldsymbol{r}(t)=(x(t), y(t)), t \in[0,1]$. Without loss of generality, we assume that $\boldsymbol{r}(t)$ is regular (i.e., $\left|\boldsymbol{r}^{\prime}(t)\right| \neq 0$ for all $t \in(0,1)$ ), and it has finite length and curvature. Recall that the parallel or offset curve generated by $\boldsymbol{r}(t)$ is defined by (cf. [19|38]):

$$
\boldsymbol{r}_{d}(t)=\boldsymbol{r}(t)+d \boldsymbol{n}(t), \quad \forall t \in[0,1]
$$

where $\boldsymbol{n}(t)$ is the unit normal to $\boldsymbol{r}(t)$ at each point, and $d$ is a preassigned signed distance. This defines a positive (exterior, $d>0$ ) or negative (interior, $d<0$ ) offset (see some examples in Figure1). For clarity of presentation, we denote the total length of $\boldsymbol{r}$ and $\boldsymbol{r}_{d}$ by $L$ and $L_{d}$, respectively. According to Lemma 3.1 in [19], the total length of $\boldsymbol{r}_{d}$ is

$$
L_{d}=\int_{0}^{1}|1+\kappa d|\left|\boldsymbol{r}^{\prime}\right| d t=\int_{0}^{L}|1+\kappa d| d s=|L+d \Delta \theta|,
$$


where $\kappa$ is the curvature and $\Delta \theta$ is the total angle of rotation of the normal $\boldsymbol{n}$ to $\boldsymbol{r}$ between $t=0$ and $t=1$, measured in the right-handed sense. Moreover, the area $A_{d}$ between the generator $\boldsymbol{r}$ and the offset $\boldsymbol{r}_{d}$ is given by

$$
A_{d}=\frac{1}{2}\left(L+L_{d}\right)|d|
$$

The interested reader may refer to 19 for detailed analysis.

Hereafter, we assume that $0<d \ll 1$, and denote the corresponding (closed) narrow band (with area $A_{d}$ ) by $R_{d}$. Recall that the total variation of the indicator function $\mathbf{1}_{R_{d}}$ characterizes the perimeter of $R_{d}$, so we deduce from (3) that

$$
T V\left(\mathbf{1}_{R_{d}}\right)=2 L+O(d), \quad 0<d \ll 1,
$$

where

$$
T V(u)=\sup _{\boldsymbol{p} \in S} \int_{\Omega} u \operatorname{div} \boldsymbol{p} d \boldsymbol{x}, \quad S:=\left\{\boldsymbol{p} \in C_{c}^{1}\left(\Omega ; \mathbb{R}^{2}\right):|\boldsymbol{p}| \leq 1\right\} .
$$

We introduce an auxiliary function $\phi$ to approximate the characteristic function:

$$
\mathbf{1}_{R_{d}} \approx \frac{1+\phi}{2}, \quad 1-\mathbf{1}_{R_{d}} \approx \frac{1-\phi}{2} .
$$

In other words, $\phi \approx 1$ if $\boldsymbol{x} \in R_{d}$ and $\phi \approx-1$ otherwise. More importantly, we enforce the constraint $\phi^{2}=1$, which acts as an intrinsic force and enables to enhance and sharpen the edges.

After collecting all the necessary facts, we present the approximation of the Mumford-Shah model (1):

$$
\min _{u, \phi^{2}=1}\left\{\frac{\alpha}{2} \int_{\Omega}(1-\phi)^{2}|\nabla u|^{2} d \boldsymbol{x}+\frac{\beta}{2} \int_{\Omega}|u-I|^{2} d \boldsymbol{x}+T V(\phi)\right\} .
$$

Compared with (2), we characterize and approximate the edge set and the length term in a different manner. The use of total variation regularization, together with the constraint, can be viewed as a sharp interface approach. It appears that the presence of TV-term and the constraint may increase the difficulty in resolving the model. To alleviate this concern, we next introduce a fast dual-type algorithm based on the Augmented Lagrangian method (ALM).

\section{Description of the Algorithm}

In this section, we introduce the algorithm to minimize the model (9). For clarity, we use boldface letters to denote vectors. As in [37, we handle the total variation term by introducing an auxiliary variable $\boldsymbol{q}$, and reformulate (9) as

$$
\min _{\substack{u, \phi^{2}=1, \boldsymbol{q}=\nabla \phi}}\left\{\frac{\alpha}{2} \int_{\Omega}(1-\phi)^{2}|\nabla u|^{2} d \boldsymbol{x}+\frac{\beta}{2} \int_{\Omega}|u-I|^{2} d \boldsymbol{x}+\int_{\Omega}|\boldsymbol{q}| d \boldsymbol{x}\right\} .
$$



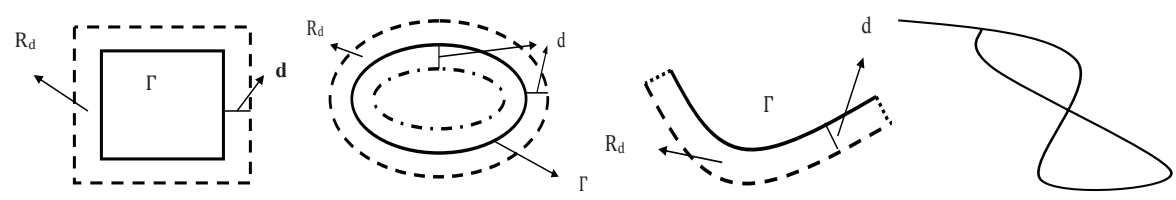

Fig. 1. The closed narrow region $R_{d}$ formed by the curve $\Gamma: \boldsymbol{r}(t)$ and its exterior parallel curve (dashed line) for closed curves and open curves (the dotted lines connected the corresponding starting points and end-points of the curve $\Gamma$ and its exterior parallel curve, respectively), where the dot-dashed line is the interior parallel curve. The last one is an example of an intersected curve that can be split into simple open or closed curves.

The augmented Lagrangian formulation for this constrained problem takes the form

$$
\begin{aligned}
\min _{u, \phi, \boldsymbol{q}} \max _{\boldsymbol{p}, \lambda}\{\mathcal{L}(u, \phi, \boldsymbol{q} ; \boldsymbol{p}, \lambda) \\
:=\frac{\alpha}{2} \int_{\Omega}(1-\phi)^{2}|\nabla u|^{2} d \boldsymbol{x}+\frac{\beta}{2} \int_{\Omega}|u-I|^{2} d \boldsymbol{x}+\int_{\Omega}|\boldsymbol{q}| d \boldsymbol{x}+(\boldsymbol{p}, \boldsymbol{q}-\nabla \phi) \\
\left.\quad+\frac{r}{2} \int_{\Omega}(\boldsymbol{q}-\nabla \phi)^{2} d \boldsymbol{x}+\frac{1}{2} \int_{\Omega} \lambda \cdot\left(\phi^{2}-1\right) d \boldsymbol{x}+\frac{r_{\phi}}{2} \int_{\Omega}\left(\phi^{2}-1\right)^{2} d \boldsymbol{x}\right\},
\end{aligned}
$$

where $\boldsymbol{p}$ and $\lambda$ are the Lagrange multipliers, and $r$ and $r_{\phi}$ are positive constants.

Thus the minimizer problem (11) is to seek a saddle point of the augmented Lagrangian functional $\mathcal{L}(u, \phi, \boldsymbol{q} ; \boldsymbol{p}, \lambda)$. But the problem (11) is not convex for both variables $\phi, u$, which means a global minimizer may not be guaranteed.

A typical approach (see, e.g., [1144]) is to split the problem (11) into several subproblems and minimize them consecutively.

It is clear that the optimality conditions for $\boldsymbol{p}$ and $\lambda$ leads to the constraint: $\boldsymbol{q}=\nabla \phi$ and $\phi^{2}=1$, respectively. Therefore, we consider the following three subproblems:

- $u$-subproblem: Given $\phi, \boldsymbol{q}$,

$$
\min _{u}\left\{\frac{\alpha}{2} \int_{\Omega}(1-\phi)^{2}|\nabla u|^{2} d \boldsymbol{x}+\frac{\beta}{2} \int_{\Omega}(u-I)^{2} d \boldsymbol{x}\right\} .
$$

- $\phi$-subproblem: Given $u, \boldsymbol{q}, \boldsymbol{p}, \lambda$,

$$
\begin{aligned}
\min _{\phi}\left\{\frac{\alpha}{2} \int_{\Omega}(1-\phi)^{2}|\nabla u|^{2} d \boldsymbol{x}+(\boldsymbol{p}, \boldsymbol{q}-\nabla \phi)+\frac{r}{2} \int_{\Omega}(\boldsymbol{q}-\nabla \phi)^{2} d \boldsymbol{x}\right. \\
\left.+\frac{1}{2} \int_{\Omega} \lambda \cdot\left(\phi^{2}-1\right) d \boldsymbol{x}+\frac{r_{\phi}}{2} \int_{\Omega}\left(\phi^{2}-1\right)^{2} d \boldsymbol{x}\right\} .
\end{aligned}
$$

- $\boldsymbol{q}$-subproblem: Given $u, \phi, \boldsymbol{p}$,

$$
\min _{\boldsymbol{q}}\left\{\int_{\Omega}|\boldsymbol{q}| d \boldsymbol{x}+(\boldsymbol{p}, \boldsymbol{q})+\frac{r}{2} \int_{\Omega}(\boldsymbol{q}-\nabla \phi)^{2} d \boldsymbol{x}\right\} .
$$


The optimality conditions for (12)-(14) yield

$$
\begin{gathered}
-\alpha \operatorname{div}\left((1-\phi)^{2} \nabla u\right)+\beta(u-I)=0, \\
\alpha(\phi-1)|\nabla u|^{2}+\operatorname{div} \boldsymbol{p}+r \operatorname{div}(\boldsymbol{q}-\nabla \phi)+\lambda \phi+2 r_{\phi}\left(\phi^{2}-1\right) \phi=0, \\
\frac{\boldsymbol{q}}{|\boldsymbol{q}|}+\boldsymbol{p}+r(\boldsymbol{q}-\nabla \phi)=0 .
\end{gathered}
$$

Since $\boldsymbol{q}=\nabla \phi$ and $\phi^{2}=1$, we find from (16) that

$$
\alpha(\phi-1)|\nabla u|^{2}+\operatorname{div} \boldsymbol{p}+\lambda \phi=0 .
$$

That is,

$$
\left(\lambda+\alpha|\nabla u|^{2}\right) \phi=-\left(\operatorname{div} \boldsymbol{p}-\alpha|\nabla u|^{2}\right) .
$$

Using the constraint $\phi^{2}=1$ to (19) again yields

$$
\left(\operatorname{div} \boldsymbol{p}-\alpha|\nabla u|^{2}\right)^{2}=\left(\lambda+\alpha|\nabla u|^{2}\right)^{2},
$$

which implies the relation between $\lambda$ and $\boldsymbol{p}$ :

$$
\lambda=-\operatorname{div} \boldsymbol{p} \quad \text { or } \quad \lambda=\operatorname{div} \boldsymbol{p}-2 \alpha|\nabla u|^{2} .
$$

Therefore, we obtain from (19) that

$$
\left.|\operatorname{div} \boldsymbol{p}-\alpha| \nabla u\right|^{2} \mid \phi=-\left(\operatorname{div} \boldsymbol{p}-\alpha|\nabla u|^{2}\right) .
$$

Since $\boldsymbol{q}=\nabla \phi$, we derive from (17) that

$$
\boldsymbol{p}=-\frac{\boldsymbol{q}}{|\boldsymbol{q}|}=-\frac{\nabla \phi}{|\nabla \phi|} \Rightarrow \nabla \phi+|\nabla \phi| \boldsymbol{p}=0 .
$$

In summary, we need to solve the system for $(u, \phi, \boldsymbol{p})$ :

$$
\left\{\begin{array}{l}
\left.|\operatorname{div} \boldsymbol{p}-\alpha| \nabla u\right|^{2} \mid \phi=-\left(\operatorname{div} \boldsymbol{p}-\alpha|\nabla u|^{2}\right), \\
\nabla \phi+|\nabla \phi| \boldsymbol{p}=0, \\
-\alpha \operatorname{div}\left((1-\phi)^{2} \nabla u\right)+\beta(u-I)=0 .
\end{array}\right.
$$

Since the binary level set function $\phi$ is expected to satisfy $\phi^{2}=1$, we adopt a MBO-type projection (see [26 36]):

$$
\phi=\mathcal{P}_{B}\left(\operatorname{div} \boldsymbol{p}-\alpha|\nabla u|^{2}\right) \text { with } \mathcal{P}_{B}(t):= \begin{cases}1, & \text { if } t \leq 0, \\ -1, & \text { if } t>0 .\end{cases}
$$

The second equation in (23) can be solved in a very similar idea in [14. For fixed $\phi$, the last linear equation for $u$ can be solved efficiently. We summarize the algorithm as follows. 


\section{Algorithm}

1. Initialization: set $\boldsymbol{p}^{0}=\mathbf{0}$ and $u^{0}=I$;

2. For $k=0,1, \cdots$,

(i) Compute

$$
\phi^{k+1}=\mathcal{P}_{B}\left(\operatorname{div} \boldsymbol{p}^{k}-\alpha\left|\nabla u^{k}\right|^{2}\right) ;
$$

(ii) Update $\boldsymbol{p}$ by the Chambolle's algorithm:

$$
\boldsymbol{p}^{k+1}=\frac{\boldsymbol{p}^{k}+\tau \nabla \phi^{k+1}}{1+\tau\left|\nabla \phi^{k+1}\right|} ;
$$

(iii) Update $u$ by

$$
u^{k+1}=u^{k}+\tilde{\tau}\left\{\alpha \operatorname{div}\left(\left(1-\phi^{k+1}\right) \nabla u^{k}\right)-\beta\left(u^{k}-I\right)\right\}
$$

3. End for till some stopping rule meets.

Some remarks are in order.

- Using the augmented Lagrangian formulation, we derive the simplified system (23), which does not depend on the parameters $r, r_{\phi}$ and $\lambda$. Hence, the algorithm might be more efficient than the algorithm, e.g., the Uzawa method, for the full model.

- The Lagrangian multiplier $\boldsymbol{p}$ turns out to be a dual variable, so the above algorithm is based on the primal-dual formulation with the complexity comparable to the fast algorithm in [14] for image denoising.

- An inner iteration can be applied to the equation for $u$, and it can be solved more efficiently other than (26). Indeed, a much deeper study can be conducted for (23), although we find the above algorithm works well.

\section{Numerical Experiments}

In this section, we provide numerical results to show the efficiency and robustness of the proposed model and algorithm. We also compare our method with Canny edge detector 11. In the numerical tests, we take $\alpha=10, \beta=1, \tau=0.12$ and $\tilde{\tau}=10^{-4}$. The stopping rule is based on the maximum pixel-wise errors $\left\|\phi^{k+1}-\phi^{k}\right\|_{\infty} \leq 10^{-2}$ and $\left\|u^{k+1}-u^{k}\right\|_{\infty} \leq 10^{-4}$.

We first test two images configured with some typical open-ended and closed curves as boundaries in Figure 2, In particular, the testing image in Figure 2 (e) consists of objects with multiple constant intensities (i.e., multi-phases). We see that in all cases, the proposed method produces much sharper edges than the commonly used Canny detector. Indeed, the binary level set function $\phi$ converges to the expected values, that is, 1 in the very narrow region surrounding 


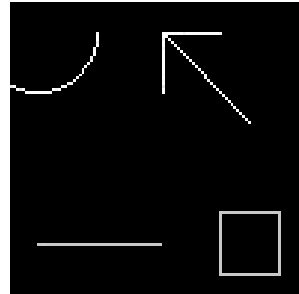

(a) Original image

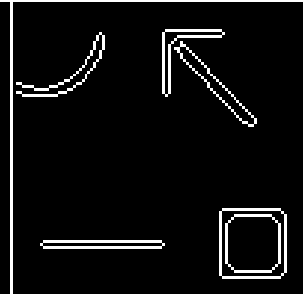

(b) Canny method

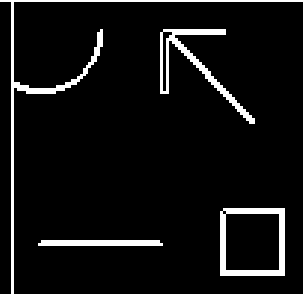

(c) Proposed method

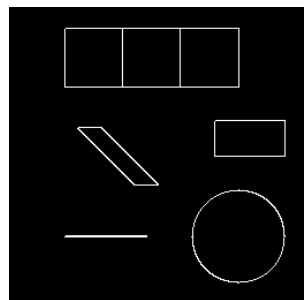

(g) Proposed method

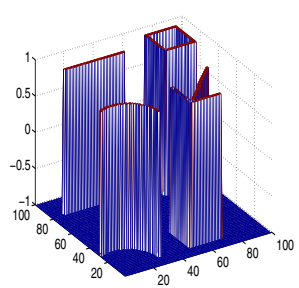

(d) $\phi$ profile

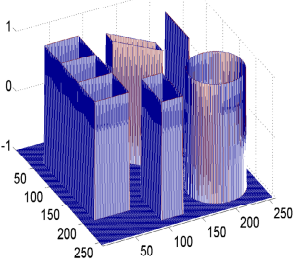

(h) $\phi$ profile

(e) Original image

(f) Canny method

Fig. 2. Two tests of edge detection by Canny method and our proposed method

the curves, and -1 elsewhere. Under the stopping criterion, our proposed algorithm takes about 30 iterations to converge. In fact, satisfactory results can usually be obtained within 10 iterations. Moreover, the algorithm is robust for the initialization and parameters. The sharp interface model together with the fast algorithm could be a very promising tool for real image processing.

Next, we test our method for images with more features. In Figure 3, we show the results of our method and compare it with the results obtained by Canny edge detector, and the Ambrosio-Tortorelli method (2). In the comparison, we take the same parameters $\alpha=10, \beta=1$ as our proposed method, and the time step is $10^{-4}$ and the $\epsilon$ in (2) is $10^{-4}$. The stopping rule is based on the maximum pixel-wise errors $\left\|v^{k+1}-v^{k}\right\|_{\infty} \leq 10^{-6}$ and $\left\|u^{k+1}-u^{k}\right\|_{\infty} \leq 10^{-6}$. Observe that our new algorithm is able to detect all the meaningful edges. More importantly, the smooth approximate solution $u$ produces a very satisfactory recovery of the original image. Once again, the outcome of the proposed method is better than that of the Canny approach. We point out that our algorithm for the Cameraman image with size 256-by-256 takes about 5 seconds with about 200 iterations to converge, while that of the Ambrosio-Tortorelli method takes more than 20 seconds and 900 iterations.

\section{Concluding Remarks}

In this paper, we propose a new approach to approximate the Mumford-Shah model for edge detection. Some features of this work are highlighted below.

- An edge is viewed as a narrow region, and a binary level set method is applied to formulate the model. Therefore, compared with the approximation in 

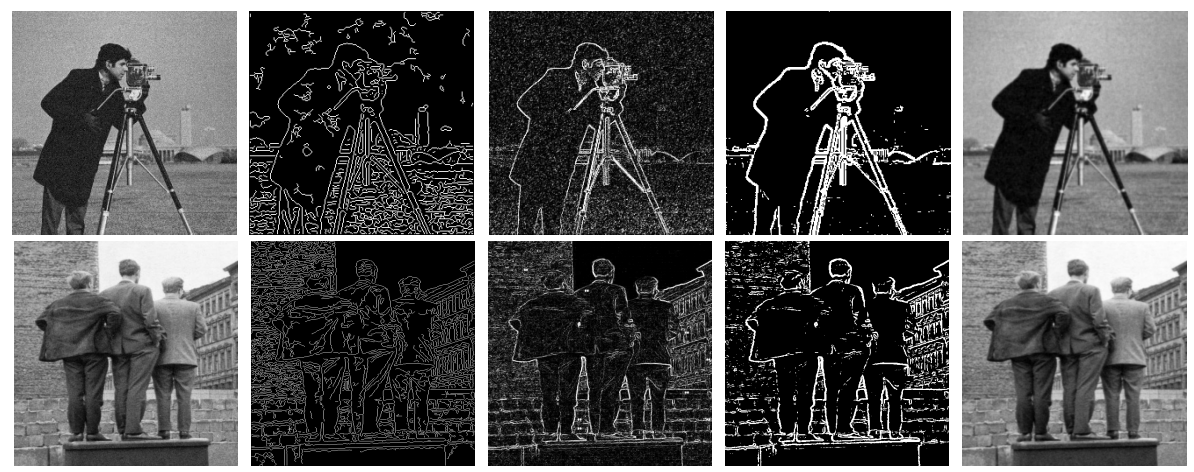

Fig. 3. Column 1: original images; Column 2: detected edges by the Canny method; Column 3: detected edges by the Ambrosio-Tortorelli method; Column 4: detected edges by our proposed method; Column 5: reconstructed image $u$ by our proposed method.

Ambrosio and Tortorelli [2, the total variation regularization is adopted to approximate the length of edge set. In general, our method can be regarded as a sharp interface approach.

- A fast primal-dual algorithm based on the augmented Lagrangian method is proposed, which contains the minimal number of parameters and is naturally initialized. The computational cost is comparable to that of the efficient algorithm in [14.

Acknowledgments. The authors would like to thank the editor and the referee for their thoughtful comments. This research is supported by Singapore MOE Grant T207B2202, and Singapore NRF2007IDM-IDM002-010. The research of the second author is also partially supported by NSFC (NO. 10801049) and Foundation of North China Electric Power University. The second author is also grateful to the hospitality of the Mathematical Image and Vision Group at the Division of Mathematical Sciences in School of Physical and Mathematical Sciences of Nanyang Technological University during the visit.

\section{References}

1. Alvarez, L., Lions, P., Morel, J.: Image selective smoothing and edge detection by nonlinear diffusion ii. SIAM J. Numer. Anal. 29(3), 845-866 (1992)

2. Ambrosio, L., Tortorelli, V.: Approximation of functions depending on jumps by elliptic functions via gamma-convergence. Comm. Pure Appl. Math. 13, 999-1036 (1990)

3. Ambrosio, L., Tortorelli, V.: On the approximation of functionals depending on jumps by quadratic, elliptic functions. Boll. Un. Mat. Ital. 6-B, 105-123 (1992)

4. Aubert, G., Kornprobst, P.: Mathematical problems in image processing: partial differential equations and the calculus of variations. Springer-Verlag, New York Inc., Secaucus (2006) 
5. Badshah, N., Chen, K.: Image selective segmentation under geometrical constraints using an active contour approach. Commun. Compu. Phys. 7(4), 759-778 (2010)

6. Bae, E., Tai, X.: Graph cut optimization for the piecewise constant level set method applied to multiphase image segmentation. In: Tai, X.-C., Mørken, K., Lysaker, M., Lie, K.-A. (eds.) SSVM 2009. LNCS, vol. 5567, pp. 1-13. Springer, Heidelberg (2009)

7. Basu, S., Mukherjee, D., Acton, S.: Implicit evolution of open ended curves. In: IEEE International Conference on Image Processing, vol. 1, pp. 261-264 (2007)

8. Berkels, B., Rätz, A., Rumpf, M., Voigt, A.: Extracting grain boundaries and macroscopic deformations from images on atomic scale. J. Sci. Comput. 35(1), 1-23 (2008)

9. Braides, A.: Approximation of free-discontinuity problems. Springer, Heidelberg (1998)

10. Brook, A., Kimmel, R., Sochen, N.: Variational restoration and edge detection for color images. J. Math. Imaging Vis. 18(3), 247-268 (2003)

11. Canny, J.: A computational approach to edge detection. IEEE Trans. Pattern. Anal. PAMI-8(6), 679-698 (1986)

12. Caselles, V., Kimmel, R., Sapiro, G.: Geodesic active contours. Int. J. Comput. Vis. 22(1), 61-79 (1997)

13. Catté, F., Lions, P., Morel, J., Coll, T.: Image selective smoothing and edge detection by nonlinear diffusion. SIAM J. Numer. Anal. 29(1), 182-193 (1992)

14. Chambolle, A.: An algorithm for total variation minimization and applications. J. Math. Imaging Vis. 20(1-2), 89-97 (2004)

15. Chan, T., Vese, L.: Active contours without edges. IEEE Trans. Image Process. 10(2), 266-277 (2001)

16. Dal Maso, G.: Introduction to $\Gamma$-convergence. Birkhauser, Basel (1993)

17. Dal Maso, G., Morel, J., Solimini, S.: A variation method in image segmentationexistence and approximation results. Acta Mathematica 168(1-2), 89-151 (1992)

18. Deriche, R.: Using canny's criteria to derive a recursively implemented optimal edge detector. Int. J. Comput. Vis. 1(2), 167-187 (1987)

19. Farouki, R., Neff, C.: Analytic properties of plane offset curves. Computer Aided Geometric Design 7(1-4), 83-99 (1990)

20. Kass, M., Witkin, A., Terzopoulos, D.: Snakes: Active contour models. Int. J. Comput. Vis. 1(4), 321-331 (1988)

21. Leung, S., Zhao, H.: A grid based particle method for evolution of open curves and surfaces. J. Comput. Phys. 228(20), 7706-7728 (2009)

22. Lie, J., Lysaker, M., Tai, X.: A binary level set model and some applications to mumford-shah image segmentation. IEEE Trans. Image Process. 15(5), 1171-1181 (2006)

23. Llanas, B., Lantaró, S.: Edge detection by adaptive splitting. J. Sci. Comput. 46(3), 486-518 (2011)

24. Ma, W., Manjunath, B.: Edgeflow: a technique for boundary detection and image segmentation. IEEE Trans. Image Process. 9(8), 1375-1388 (2000)

25. Meinhardt, E., Zacur, E., Frangi, A., Caselles, V.: 3D edge detection by selection of level surface patches. J. Math. Imaging Vis. 34(1), 1-16 (2009)

26. Merriman, B., Bence, J., Osher, S.: Motion of multiple functions: a level set approach. J. Comput. Phys. 112(2), 334-363 (1994)

27. Mumford, D., Shah, J.: Optimal approximations by piecewise smooth functions and associated variational problems. Comm. Pure Appl. Math 42(5), 577-685 (1989) 
28. Osher, S., Sethian, J.: Fronts propagating with curvature dependent speed: Algorithms based on hamilton-jacobi formulations. J. Comput. Phys. 79(1), 12-49 (1988)

29. Paragios, N., Chen, Y., Faugeras, O.: Handbook of mathematical models in computer vision. Springer-Verlag New York Inc., Secaucus (2006)

30. Perona, P., Malik, J.: Scale-space and edge-detection using anisotropic diffusion. IEEE Trans. Pattern. Anal. 12(7), 629-639 (1990)

31. Pock, T., Cremers, D., Bischof, H., Chambolle, A.: An algorithm for minimizing the Mumford-Shah functional. In: 12th International Conference on Computer Vision, pp. 1133-1140. IEEE, Los Alamitos (2009)

32. Smereka, P.: Spiral crystal growth. Physica D: Nonlinear Phenomena 138(3-4), 282-301 (2000)

33. Smith, S.: Edge thinning used in the susan edge detector. Technical Report, TR95SMS5 (1995)

34. Sun, Y., Wu, P., Wei, G., Wang, G.: Evolution-operator-based single-step method for image processing. Int. J. Biomed. Imaging, 1-28 (2006)

35. Suzuki, Y., Takayama, T., Motoike, I., Asai, T.: A reaction-diffusion model performing stripe-and spot-image restoration and its lsi implementation. Electronics and Communications in Japan (Part III: Fundamental Electronic Science) 90(1), 20-29 (2007)

36. Tai, X., Christiansen, O., Lin, P., Skjælaaen, I.: Image segmentation using some piecewise constant level set methods with MBO type of projection. International Journal of Computer Vision 73(1), 61-76 (2007)

37. Tai, X.C., Wu, C.: Augmented lagrangian method, dual methods and split bregman iteration for rof model. In: Tai, X.-C., Mørken, K., Lysaker, M., Lie, K.-A. (eds.) SSVM 2009. LNCS, vol. 5567, pp. 502-513. Springer, Heidelberg (2009)

38. Toponogov, V.: Differential geometry of curves and surfaces: a concise guide. Birkhauser, Basel (2006)

39. Upmanyu, M., Smith, R., Srolovitz, D.: Atomistic simulation of curvature driven grain boundary migration. Interface Sci. 6, 41-58 (1998)

40. Vese, L., Chan, T.: A multiphase level set framework for image segmentation using the mumford and shah model. Int. J. Comput. Vis. 50(3), 271-293 (2002)

41. Wang, Y., Yang, J., Yin, W., Zhang, Y.: A new alternating minimization algorithm for total variation image reconstruction. SIAM J. Imaging Sci. 1(3), 248-272 (2008)

42. Wei, G., Jia, Y.: Synchronization-based image edge detection. EPL (Europhysics Letters) 59(6), 814-819 (2002)

43. Witkin, A.P.: Scale-space filtering. In: Proc. 8th Int. Joint Conf. Art. Intell., Karlsruhe, Germany, pp. 1019-1022 (1983)

44. Wu, C., Zhang, J., Tai, X.: Augmented lagrangian method for total variation restoration with non-quadratic fidelity. In: UCLA, CAM09-82, pp. 1-26 (2009) 